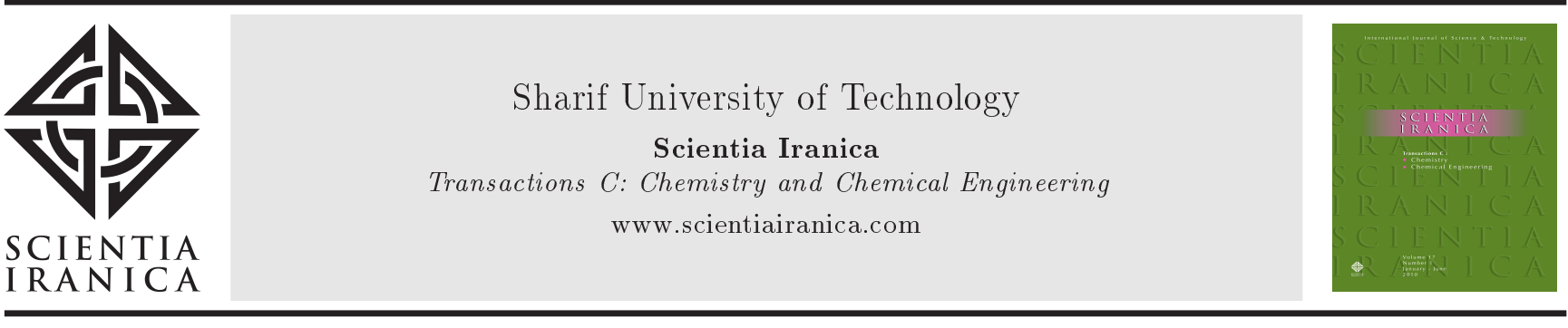

\title{
Study of self-diffusion in two binary solutions, glycerol-water and methanol-water using diffusion-ordered spectroscopy
}

\author{
E. Fadaei and M. Tafazzoli* \\ Department of Chemistry, Sharif University of Technology, Tehran, P.O. Box 11155-9516, Iran.
}

Received 9 August 2015; received in revised form 10 December 2015; accepted 3 January 2016

KEYWORDS
Self-diffusion;
DOSY NMR;
Glycerol;
Methanol;
Concentration effect;
Temperature effect.

\section{Introduction}

The aqueous solutions of alcohols such as methanol, ethylene glycol, and glycerol are commonly used as cryoprotectant to protect biological molecules, cells, and tissues from the freezing damages [1-3].

Methanol is also well-known as an amphiphilic molecule which has both hydrophobic $\left(\mathrm{CH}_{3}\right)$ and hydrophilic $(\mathrm{OH})$ groups and it is suitable for studying the interaction of the proteins in the biological systems [4].

Different computational and experimental methods have previously been used to study the molecular structure of the alcohol solutions. These methods include molecular dynamics [5], neutron diffraction [6],

*. Corresponding author. Fax: +982166029165 E-mail addresses: e_fadaei@ch.sharif.edu (E. Fadaei); tafazzoli@sharif.edu (M. Tafazzoli) infrared spectroscopy [7], Raman spectroscopy [4], terahertz time-domain spectroscopy [8], dielectric spectroscopy [9], and nuclear magnetic resonance spectroscopy [10]. In spite of numerous studies, the dynamical and structural properties of alcohol solutions are not still entirely understood. From previous results, it is obvious that the influence of the alcohol on the hydrogen bonding network of water plays a key role in the physicochemical properties of these solutions $[5,11$ $15]$.

Self-diffusion of molecules is due to their random translational motions which are driven by internal kinetic energy [16]. This type of diffusion takes place in the absence of concentration (or chemical potential) gradient. Since self-diffusion coefficient of a single molecule is related to the molecular structure and solute-solvent interactions, it is a significant physicochemical property which helps 
to characterize the molecular structure of solutions or pure liquids [17-19]. Various computational and experimental methods, such as molecular dynamics simulation [5], Taylor dispersion [20,21], interferometric microdiffusion [20], quasi-elastic neutron scattering [22,23], diaphragm-cell technique [24], and NMR spectroscopy $[16,25]$, have been used to measure the diffusion coefficient.

Diffusion-Order Spectroscopy (DOSY) is a useful NMR experiment which measures the self-diffusion coefficient of each component in the mixture, separately [26]. The self-diffusion coefficient $(D)$ obtained by this technique is described, theoretically, with Stokes-Einstein equation (Eq. (1)) [16]:

$$
D=\frac{k_{B} T}{6 \pi \eta r_{s}}
$$

where $k_{B}$ is the Boltzmann constant, $T$ the temperature, $\eta$ the viscosity of the solution, and $r_{s}$ the hydrodynamic radius of the molecule.

Temperature and concentration are two principal factors that influence the self-diffusion coefficient. In the previous research, the role of the mentioned factors has been studied in the aqueous solutions $[9,11,20,27$ 29]. According to the previous works, the dynamic behavior of water and alcohol in their aqueous mixtures strongly depends on the concentrations of alcoholwater solutions. The alcohol systems have various behaviors in different ranges of concentration.

Towey and Dougan have investigated the glycerol solutions in both low- and high-concentration ranges. Their results indicate that, in the dilute solution, the hydrogen bond network is highly mixed between glycerol and water [6]. Moreover, in the concentrated solutions, the water-water hydrogen bonds are significantly perturbed and water molecules take the place of the glycerol molecules in their network. Also, there are a number of studies which investigate the structure of water-methanol binary mixtures in different concentration ranges. $\mathrm{Li}$ et al. have identified three critical composition ranges for studying the molecular structure of alcohol solution. They found that methanol solution has different structures at mole fractions of $0.1,0.3$, and $0.7[8]$.

However, the effect of concentration and temperature on the self-diffusion of components in waterglycerol and water-methanol mixtures has not been extensively studied, experimentally.

In this work, we examined the molecular behavior of the aqueous solution of glycerol as a sugar alcohol and methanol as an amphiphilic molecule at different concentrations and temperatures with measuring the self-diffusion coefficient using DOSY NMR experiment.

The present results provide insights into the mechanism of the dependence of water and alcohol self- diffusion on temperature and concentration of aqueous binary mixtures.

\section{Materials and methods}

Glycerol and methanol were supplied by Merck Millipore. Methanol-water solutions were prepared in methanol mole fractions of $0.03,0.1$, and 0.2 . Glycerolwater solutions were prepared in glycerol mole fractions of $0.008,0.03,0.1$, and 0.2 . $\quad \mathrm{D}_{2} \mathrm{O}$ (purity>99.8) was used as solvent in solution preparation for NMR measurements.

The self-diffusion coefficients were measured by diffusion-order NMR spectroscopy. All ${ }^{1} \mathrm{H}$ DOSY experiments were carried out on a Bruker Avance500 spectrometer. The pulse sequence of longitudinal eddy current delay with 2 spoil bipolar gradient pulses (ledbpgp2s) [30] was used for all samples. The eddy current and the gradient recovery delay were kept at fixed values of 5 and $0.1 \mathrm{~ms}$, respectively. The pulse field gradient duration $(\delta)$ varied from 1.8 to $2 \mathrm{~ms}$. The diffusion period $(\Delta)$ varied from 70 to $100 \mathrm{~ms}$. The strength of the pulse field gradient increased, gradually, from 2 to $95 \%$ of maximum value in 16 steps. The parameters $\Delta$ and $\delta$ were optimized so that the intensity of signals can decrease one order of magnitude when field gradient increases from $2 \%$ to $95 \%$. The signal exponential decay is described by the StejskalTanner expression:

$$
I=I_{0} \exp \left(-D q^{2}\left(\Delta-\frac{\delta}{3}\right)\right),
$$

where $I$ is the signal intensity (or integral) and $I_{0}$ is the initial signal intensity (or integral); $q=\gamma_{H} \delta G$, where $\gamma_{H}$ is the magnetogyric ratio for ${ }^{1} H$ and $G$ is the gradient magnitude $[31,32]$. The temperature of the sample was carefully controlled with a Bruker BT-3000 unit. It was kept at $298.0 \mathrm{~K}$ in Section 3.1 to study the concentration effect, and it varied from 293.0 to 308.0 $\mathrm{K}$ in Section 3.2 to study the temperature effect on the self-diffusion coefficients.

The self-diffusion coefficients of glycerol and methanol were measured by following $\mathrm{CH}_{2}$ and $\mathrm{CH}_{3}$ NMR signals at chemical shifts of 3.68 and 3.17 , respectively. Also, the self-diffusion coefficient of water was determined by following the NMR signal of $\mathrm{OH}$ protons at chemical shifts of 4.72 and 4.76 for glycerol and methanol solutions, respectively. All data processing and analyzing were done with XWINNMR (Beta version 3.5). Finally, $\log D$ of each component was obtained from the DOSY map.

In order to confirm correctness of our results, the logarithm of water diffusion coefficient $(\log D)$ measured by DOSY experiment was compared with the corresponding data reported in the previous literature [33]; the uncertainty was $0.14 \%$. 


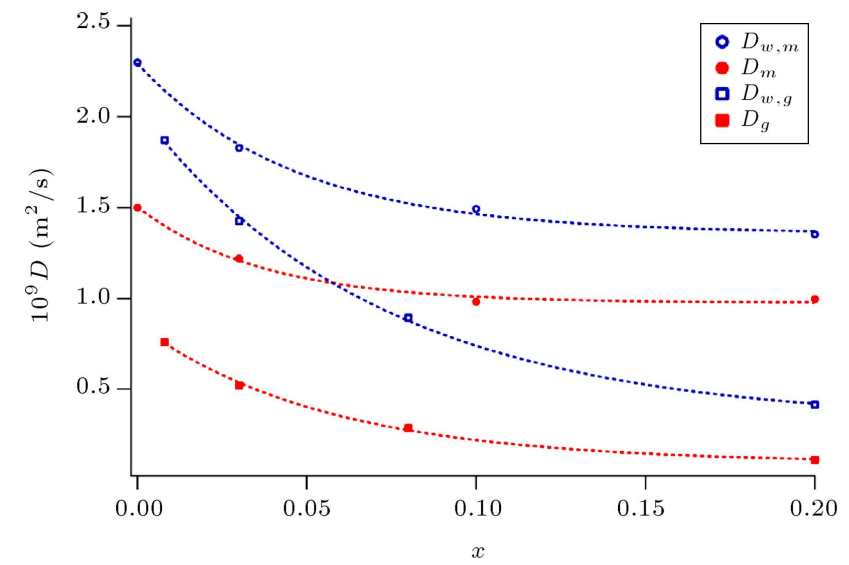

Figure 1. Self-diffusion coefficients versus mole fraction of alcohols. Water self-diffusion coefficient in glycerol-water solutions, $D_{w, g}$, glycerol self-diffusion coefficient in glycerol-water solutions, $D_{g}$, water self-diffusion coefficient in methanol-water solutions, $D_{w, m}$, and methanol self-diffusion coefficient in methanol-water solutions, $D_{m}$. The data at $x=0$ for methanol solution was calculated from Eq. (3). The dashed lines show interpolation of the experimental data (Eq. (3)).

\section{Results and discussion}

\subsection{Concentration dependence of the self-diffusion coefficient}

In order to examine concentration effect on molecular behavior of the alcohol solutions, the self-diffusion coefficients of water $\left(D_{w}\right)$, glycerol $\left(D_{g}\right)$, and methanol $\left(D_{m}\right)$ were measured in binary mixtures of glycerolwater $(0.008,0.03,0.08$, and 0.2 glycerol mole fractions) and methanol-water $(0.03,0.1$, and 0.2 methanol mole fractions).

Figure 1 depicts the variation of self-diffusion coefficients of water and alcohols versus mole fraction of alcohol, $x$, in the solutions mentioned above. The self-diffusion coefficients were measured using DOSY NMR experiments at $298.0 \mathrm{~K}$.

The experimental data of the concentration behavior of both glycerol and methanol solutions was well fitted by Eq. (3) (Figure 1) which had been before by D'Errico et al. [20] to describe concentration dependence of the diffusion coefficient:

$$
D=\frac{A-B x}{1+C x},
$$

$D$ is the self-diffusion coefficient of the components (glycerol, methanol, or water) and $x$ is the mole fraction of alcohol. $A, B$, and $C$ are the equation constants.

Apart from magnitude, the variation of the selfdiffusion coefficient of water and alcohols has a similar trend. The self-diffusion coefficients decrease as mole fraction of alcohol increases. This trend indicates that there is a significant interaction between water and

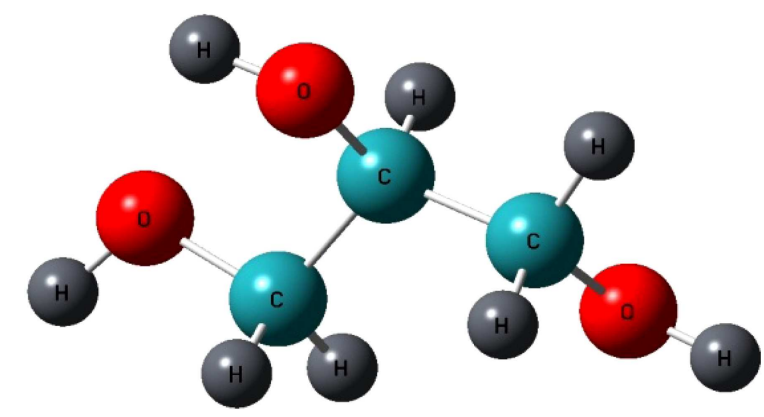

Figure 2. 3D exhibition of glycerol molecular structure.

alcohol that causes the self-diffusion of both water and alcohol to decrease. This trend has also been reported in previous studies $[8,20,34]$.

According to the previous research, three types of hydrogen bonds can be formed in the wateralcohol solutions: water-water (w-w), water-alcohol (w-s), and alcohol-alcohol (s-s) hydrogen bonds. In dilute methanol and glycerol solutions studied in this work, the w-w and w-s hydrogen bonds are the most important kinds of hydrogen bonds [6]. Therefore, we should concentrate on these kinds of the hydrogen bonds to understand how the hydrogen bonds influence the molecular behavior of glycerol-water and methanolwater solutions.

\subsubsection{Behavior of the glycerol solution}

As seen in Figure 1, the water and glycerol self-diffusion coefficient decreases sharply in the lower glycerol mole fractions. It depicts the existence of strong bonds between water and glycerol which retards the molecular diffusion.

As seen in Figure 2, the glycerol molecule has three hydroxyl groups. According to the computational analysis, these hydroxyl groups are capable to form a maximum of six hydrogen bonds [6].

Glycerol molecules with three hydroxyl groups can attract water molecules in their hydrogen bonding sites. As a result, water self-diffusion is controlled by glycerol diffusion, and water molecules slow down very fast. This trend is also observed for glycerol. It seems that glycerol has also tendency to bond with water molecules, strongly. According to the findings of Towey and Dougan [6], glycerol molecules have tendency to form hydrogen bonds with water molecules more than themselves. Towey and Dougan have compared the number of hydrogen bonds per water and glycerol molecules in dilute solutions with that in their pure liquids. They have asserted that the number of waterglycerol hydrogen bonds compensates for reduction in the water-water hydrogen bonds in the dilute solutions. Therefore, the number of hydrogen bonds per water molecules remains constant ( 3.7 hydrogen bonds). In other words, the glycerol molecules can participate in the water hydrogen bond network like water molecules. 
Also, the number of hydrogen bonds formed by glycerol molecules in dilute solution (6.3 hydrogen bonds) is larger than that in pure glycerol liquid ( 5.7 hydrogen bonds). It is a consequence of a large number of possible conformations of glycerol in the dilute solution. Indeed, the backbone of the glycerol molecule can move freely in the dilute solution and it creates various conformers [6]. Therefore, the glycerol molecules are able to find suitable conformers to bond with the maximum number of water molecules. The high tendency of the water and glycerol to form hydrogen bonds causes the water molecules to be kept by glycerol tightly. This makes the water and glycerol molecules move hardly. As a result, the self-diffusion coefficients decrease as the glycerol concentration increases.

As seen in Figure 1, the self-diffusion coefficient of water and glycerol decreases with a slighter slope where concentration increases up to the mole fraction of 0.20 . It seems that the hydrogen bonds between water and glycerol change in higher concentrations. Glycerol has three hydroxyl groups and it is capable to form six hydrogen bonds [9]. The hydrogen-bonding sites could be occupied entirely by water molecules in more diluted solutions, because there are a large number of free water molecules which can occupy glycerol binding sites. As concentration increases, some of the hydrogen-bonding sites of the glycerol remain unoccupied (or may be occupied by glycerol molecules). Thereafter, the water-water and waterglycerol hydrogen bonds are affected by concentration, weakly, when the solution becomes concentrated (up to $x=0.20$ ). As a result, $D_{w}$ and $D_{g}$ decrease with a slighter slope in higher concentration rather than those in dilute solutions. Additionally, the molecular dynamics simulations have shown that the water-water hydrogen bonding lifetime increases when glycerol mole fraction increases [5]; as a consequence, the water molecules remain in their hydrogen bonds network for a longer time. They are not widely available for the glycerol molecules in concentrated solutions (about 0.20 mole fraction of glycerol) compared to very dilute solutions (about 0.03 mole fraction of glycerol). It also causes a fewer number of hydrogen bonds per glycerol. It makes $D_{w}$ and $D_{g}$ decrease slower in higher concentrations.

Another point in Figure 1 is that $D_{w}$ tends to $D_{g}$ in the concentrated solutions. As mole fraction of glycerol increases, a point is reached, in which glycerol molecules attract all of the water molecules on their hydrogen bonding sites. At this point, the number of water molecules is fewer than the number of glycerol hydrogen bonding sites. In these conditions, there are no effective free water molecules and all of them are bonded to glycerol. Thus, water diffusion will be controlled by glycerol, entirely. As we expect, the larger molecules diffuse slower than molecules with smaller sizes (Eq. (1)). Therefore, the self-diffusion coefficient of water decreases to the self-diffusion coefficient of glycerol. This trend has also been observed in the case of concentrated solutions (upper than $x=$ $0.2)[20,28]$. The formation of glycerol clusters should also be noticed for study of the more concentrated solutions $[10,28]$.

\subsubsection{Behavior of the methanol solution}

Methanol is an amphiphilic molecule which has a hydrophobic head more than its hydrophilic head. Therefore, the methanol interactions in the aqueous mixture are not easy to be understood. Methanol is capable to form 1:1 compound through the hydrogen bonding interactions with water, and affects the water network, weakly. This interaction impacts just on the first hydration shell and does not extend to the bulk [12]. Also, the methanol molecules are strongly solvated by a cage of water due to the hydrophobic forces [34]. However, in low concentrations, there is a slight preference for hydrophilic interactions through the hydrogen bonds. Additionally, since the water molecules are enough in the dilute solutions, the hydration shell would be formed and the methanol clusters cannot be formed [34]. Hence, the hydrogen bonds between water and methanol are the major kind of interactions which interpret the behavior of the system.

As seen in Figure $1, D_{m}$ is larger than $D_{g}$ at all concentrations. It is a consequence of smaller size and less steric hindrance of the methanol than those of glycerol that helps it to diffuse faster according to Stokes-Einstein equation (Eq. (1)). In the case of water self-diffusion, the number of water molecules, which are bonded to glycerol in the glycerol-water solution, is much more than those bonded to methanol in the methanol-water solution. Therefore, the water molecules motions are more retarded by alcohol molecules in glycerol-water solution than in methanolwater solution. Hence, the water self-diffusion coefficient exponentially dips and converges to glycerol selfdiffusion coefficient faster than methanol self-diffusion coefficient.

\subsection{Temperature dependence of the self-diffusion coefficient}

In order to further examine the molecular behavior of glycerol and methanol solutions, the temperature effect was studied by measuring the self-diffusion coefficients of water, glycerol, and methanol in glycerol-water and methanol-water solutions (with alcohol mole fraction of 0.03) using DOSY NMR; temperature varied from 293.0 to $308.0 \mathrm{~K}$.

As shown in Figure 3, the self-diffusion coefficients increase as the temperature increases. This result is consistent with Stokes-Einstein equation (Eq. (1)). Based on this relation, the self-diffusion coefficient 


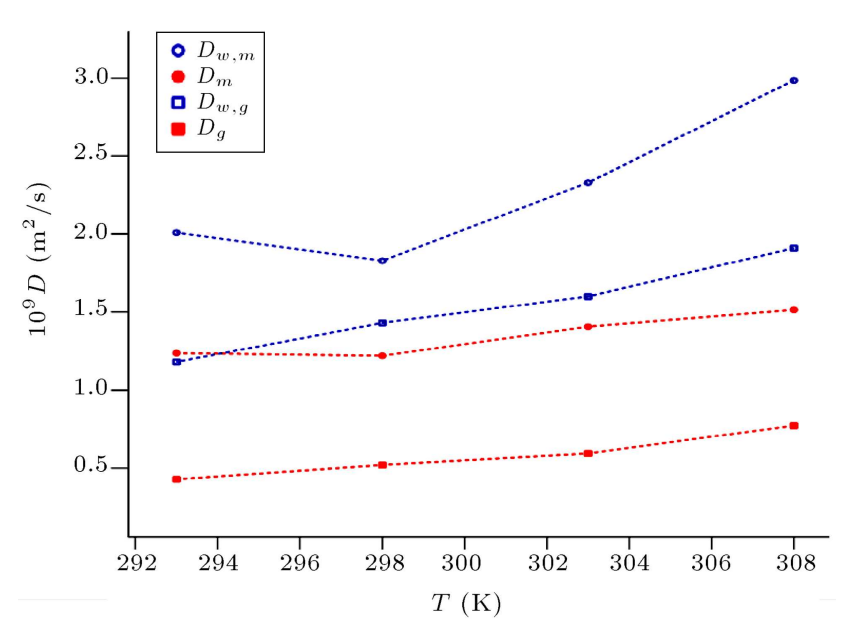

Figure 3. Temperature dependence of the self-diffusion coefficient. Water self-diffusion coefficient in glycerol-water solutions, $D_{w, g}$, glycerol self-diffusion coefficient in glycerol-water solutions, $D_{g}$, water self-diffusion coefficient in methanol-water solutions, $D_{w, m}$, and methanol self-diffusion coefficient in methanol-water solutions, $D_{m}$. The dashed lines are just used to guide the eyes.

is larger in higher temperatures. As expected, the thermal motions of the molecules increase as the temperature rises.

Although the self-diffusion deviates from the Stokes-Einstein relation in some cases, it obeys the relation in the temperature ranges studied in this work (above $290 \mathrm{~K}$ ) [35].

Moreover, the lifetimes of the w-w and w-s hydrogen bonds (time that two molecules remain continuously hydrogen bonded) decrease as temperature increases [5]. It confirms that the molecules break down their hydrogen bonds faster and release them from the hydrogen bond network easier with temperature rising. Thus, the self-diffusion coefficients increase as temperature increases.

The measured self-diffusion coefficients show an Arrhenius temperature behavior as:

$$
D=D_{0} \exp \left(-\frac{E_{a}}{R T}\right),
$$

where $E_{a}$ is activation energy of self-diffusion, $R$ is the gas constant equal to $8.314(\mathrm{~J} / \mathrm{mol} . \mathrm{K})$, and $D_{0}$ is a parameter allocated to the pre-exponential factor in Arrhenius relation. This temperature behavior has also been reported for diffusion in other similar solutions like ethanol [36]. Figure 4 shows interpolation of our experimental data according to the natural logarithm of Arrhenius equation (Eq. (4)).

Approximate values of self-diffusion activation energy of water and alcohols in glycerol-water and methanol-water (with 0.03 mole fraction of alcohol) were extracted from Eq. (4). The estimated values are summarized in Table 1.

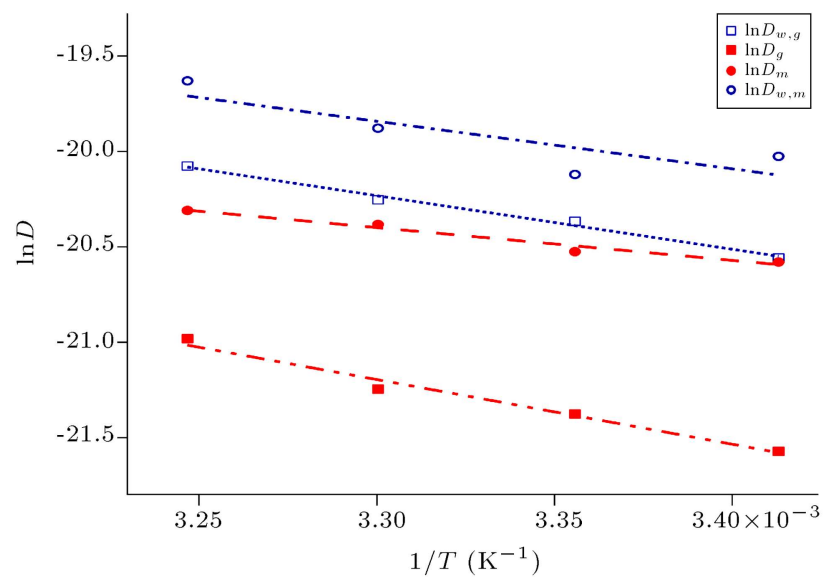

Figure 4. $\ln D$ versus $1 / T$. Water self-diffusion coefficient in glycerol-water solutions, $D_{w, g}$, glycerol self-diffusion coefficient in glycerol-water solutions, $D_{g}$, water self-diffusion coefficient in methanol-water solutions, $D_{w, m}$, and methanol self-diffusion coefficient in methanol-water solutions, $D_{m}$. The lines show interpolation of experimental data (Eq. (4)).

Table 1. Self-diffusion activation energies of alcohol $\left(E_{a, s}\right)$ and water $\left(E_{a, w}\right)$ in glycerol-water and methanol-water solutions (with 0.03 mole fraction of alcohol) obtained by curve fitting (Eq. (4)).

\begin{tabular}{ccc}
\hline Solution & $\boldsymbol{E}_{\boldsymbol{a}, \boldsymbol{s}} / \mathbf{k J ~} \mathbf{~ m o l}^{-\mathbf{1}}$ & $\boldsymbol{E}_{\boldsymbol{a}, \boldsymbol{w}} / \mathbf{k J} \mathbf{~ m o l}^{-\mathbf{1}}$ \\
\hline Glycerol-water & $28.0 \pm 2.0$ & $23.0 \pm 1.0$ \\
Methanol-water & $13.0 \pm 3.0$ & $25.0 \pm 0.9$ \\
\hline
\end{tabular}

As seen in Table 1, the self-diffusion activation energy values of water in two solutions are almost close to each other. This result suggests the same mechanism for water diffusion in two solutions. In contrast, the self-diffusion activation energy of methanol is significantly lower than that of glycerol. It suggests a different mechanism for methanol diffusion.

\subsubsection{Temperature behavior of the methanol solution in different concentrations}

In order to further explore the reasons of the different temperature behaviors of methanol, we also investigate the temperature effect on the methanol solutions with different concentrations (0.03 to 0.20 mole fractions of methanol). The self-diffusion coefficients measured using DOSY NMR are summarized in Table 2.

The Arrhenius plots of the measured self-diffusion coefficients are illustrated in Figure 5 and activation energies are summarized in Table 3.

$E_{a, w}$ is approximately the same in all solutions and it just increases by $11 \%$, while $E_{a, m}$ increases by $148 \%$ as mole fraction of methanol increases from 0.03 to 0.20 . This result depicts that water diffuses in the same mechanism in three solutions but methanol has a different behavior.

As seen in Table 3, the self-diffusion activation en- 
Table 2. Self-diffusion coefficients of methanol $\left(D_{m}\right)$ and water $\left(D_{w}\right)$ in the methanol-water solutions (with $x_{m}$ mole fraction of methanol) at different temperatures.

\begin{tabular}{|c|c|c|c|c|c|c|}
\hline \multirow[b]{2}{*}{$T(\mathbf{K})$} & \multicolumn{2}{|c|}{$x_{m}=0.03$} & \multicolumn{2}{|c|}{$x_{m}=0.10$} & \multicolumn{2}{|c|}{$x_{m}=0.20$} \\
\hline & $10^{9} D_{m} / \mathrm{m}^{2} \mathrm{~s}^{-1}$ & $10^{9} D_{w} / \mathrm{m}^{2} \mathrm{~s}^{-1}$ & $10^{9} D_{m} / \mathrm{m}^{2} \mathrm{~s}^{-1}$ & $10^{9} D_{w} / \mathrm{m}^{2} \mathrm{~s}^{-1}$ & $10^{9} D_{m} / \mathrm{m}^{2} \mathrm{~s}^{-1}$ & $10^{9} D_{w} / \mathrm{m}^{2} \mathrm{~s}^{-1}$ \\
\hline 293.0 & 1.155 & 1.717 & 0.923 & 1.406 & 0.817 & 1.153 \\
\hline 298.0 & 1.219 & 1.828 & 0.982 & 1.493 & 0.995 & 1.352 \\
\hline 303.0 & 1.406 & 2.328 & 1.265 & 1.836 & 1.225 & 1.493 \\
\hline 308.0 & 1.514 & 2.985 & 1.656 & 2.339 & 1.611 & 2.080 \\
\hline
\end{tabular}

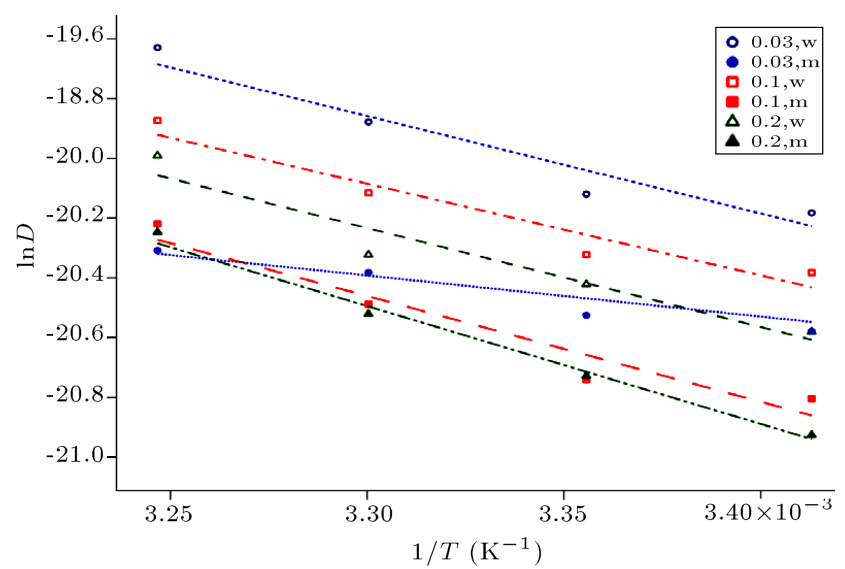

Figure 5. $\ln D$ versus $1 / T$ for methanol (solid markers) and water (open markers) in methanol-water solutions with methanol mole fractions of 0.03 (circles), 0.10 (squares), and 0.20 (triangles). Lines show interpolation of the experimental data (Eq. (4)).

Table 3. Self-diffusion activation energy of methanol $\left(E_{a, m}\right)$ and water $\left(E_{a, w}\right)$ in methanol-water solutions (with different methanol mole fractions) obtained by curve fitting (Eq. (4)).

\begin{tabular}{ccc}
$\begin{array}{c}\text { Methanol } \\
\text { mole } \\
\text { fraction }\end{array}$ & $\boldsymbol{E}_{\boldsymbol{a}, \boldsymbol{m}} / \mathbf{k J} \mathbf{~ m o l}^{-\mathbf{1}}$ & $\boldsymbol{E}_{\boldsymbol{a}, \boldsymbol{w}} / \mathbf{k} \mathbf{J} \mathbf{~ m o l}^{-\mathbf{1}}$ \\
\hline 0.03 & $13.606 \pm 0.029$ & $24.811 \pm 0.087$ \\
0.10 & $29.431 \pm 0.066$ & $25.540 \pm 0.057$ \\
0.20 & $32.783 \pm 0.028$ & $27.552 \pm 0.064$ \\
\hline
\end{tabular}

ergy of methanol is significantly low $\left(13.606 \mathrm{~kJ} \mathrm{~mol}^{-1}\right.$ ) in very dilute solution (mole fraction of 0.03 ). It shows that methanol diffuses freely in this concentration. It can be concluded that diffusion mechanism of methanol molecules in this composition is different from those of the other two solutions.

The water-methanol hydrogen bonds in diluted solutions are stronger than water-water hydrogen bonds [37]. As seen in Table 3, while the self-diffusion activation energy of methanol is more than those of water solutions with 0.1 and 0.2 mole fractions of methanol, this value is smaller than that of water in the solution with 0.03 mole fraction of methanol. This data confirms that hydrogen bonding network in the solution with 0.03 mole fraction is completely different from those in the other two solutions. It seems that water-methanol hydrogen bonds are not formed significantly in the very dilute solution. Although numerous studies have investigated the diffusion mechanism of methanol in very dilute solutions, it is not still entirely understood. Lin et al. have suggested four different mechanisms depending on the concentration range of the alcohol solutions. For very dilute methanol solution (below the mole fraction of 0.1), they have confirmed the formation of iceberg-like as a possible mechanism to interpret the unusual behavior of methanol [4]. In this mechanism, the water molecules, around methanol, form hydrogen bonds with themselves because of hydrophobic effect of $\mathrm{CH}_{3}$ group of methanol molecules. This will free up space for methanol to diffuse more freely and more quickly. As a result, the self-diffusion activation energy of methanol is significantly low in very dilute solutions (0.03 mole fraction of methanol solution studied in this work) (Table 3). Corsaro et al. have also confirmed that water molecules in very diluted solutions prefer to make hydrogen bond with themselves rather than methanol molecules. This conclusion also confirms the easy and fast diffusion of methanol molecules [38].

For other water-methanol solutions (with 0.1 and 0.2 methanol mole fractions), the structural properties of methanol solutions can be explained by hydrogen bonding. As mentioned before, in dilute solutions, methanol molecules form hydrogen bonds which affect only the first hydration shell of the water, and the tetrahedral structure of water remains constant in the bulk. Therefore, the water molecules diffuse approximately in the same manner in different solutions. Otherwise, the methanol molecules tend to form much more hydrogen bonds with water molecules when the mole fraction of methanol increases. In more concentrated solutions, the structure of water molecules is disrupted slightly. This small perturbation occurs in the water network as much as the first hydration shell is formed around the methanol molecules. As a result, thermal motions of the methanol are retarded and $E_{a, m}$ increases. At very low temperatures, the methanol clusters may be formed. This matter should be noticed to investigate the structure of the solutions at lower temperatures [13]. The molecular behavior 
of water and alcohols in aqueous binary mixture at very low temperatures is important in the case of their cryoprotection applications. However, study of the molecular dynamics from the hydrogen bonding viewpoint is valuable in other different applications of these solutions.

\section{Conclusion}

In this study, the effect of concentration and temperature on the self-diffusion coefficient of water, glycerol, and methanol in glycerol-water and methanol-water solutions was investigated by DOSY NMR experiment. Decreasing the self-diffusion coefficients of both water and alcohol as mole fraction of alcohol increases indicates that there are hydrogen bond interactions between water and alcohol, which cause slower diffusion for both. In the case of glycerol-water solution, the larger number of water molecules bonded to glycerol causes a sharper decline in the self-diffusion coefficient in very dilute glycerol solutions than in concentrated solutions. It also causes a steeper decrease of the selfdiffusion coefficient of glycerol than that of methanol, which can form one hydrogen bond with water.

Self-diffusion coefficients increase as temperature rises, which is due to temperature dependence of molecules' thermal motions. In the case of methanolwater solution, the temperature analyses also show that in the dilute solutions (studied in this work), water diffuses in the same manner, but methanol's mechanism varies in different concentrations. The estimated activation energy of the self-diffusion provides insights into the hydrogen bond network of the methanol and water in methanol-water solutions. Our findings are consistent with previous studies, which suggest the formation of iceberg-like structure in the methanolwater solution. This structure helps methanol to diffuse faster in the very dilute solution used in this study (0.03 mole fraction of methanol).

\section{Acknowledgment}

The authors are grateful to the research vice-president of Sharif University of Technology for financial support. Also, they appreciate IDB for providing the grant for purchasing the NMR instrument.

\section{References}

1. Pagliaro, M. and Rossi, M. "Glycerol: Properties and production", The Future of Glycerol, pp. 20-21 (2010).

2. Lahnsteiner, F., Weismann, T. and Patzner, R.A. "Methanol as cryoprotectant and the suitability of $1.2 \mathrm{ml}$ and $5 \mathrm{ml}$ straws for cryopreservation of semen from salmonid fishes", Aquaculture Research, 28(6), pp. 471-479 (1997).
3. Dashnau, J.L., Nucci, N.V., Sharp, K.A. and Vanderkooi, J.M. "Hydrogen bonding and the cryoprotective properties of glycerol/water mixtures", The Journal of Physical Chemistry B, 110(27), pp. 1367013677 (2006).

4. Lin, K., Hu, N., Zhou, X., Liu, S. and Luo, Y. "Reorientation dynamics in liquid alcohols from Raman spectroscopy", Journal of Raman Spectroscopy, 43(1), pp. $82-88$ (2012).

5. Zhang, N., Li, W., Chen, C., Zuo, J. and Weng, L. "Molecular dynamics study on water self-diffusion in aqueous mixtures of methanol, ethylene glycol and glycerol: investigations from the point of view of hydrogen bonding", Molecular Physics, 111(7), pp. 939-949 (2013).

6. Towey, J.J. and Dougan, L. "Structural examination of the impact of glycerol on water structure", The Journal of Physical Chemistry B, 116(5), pp. 16331641 (2011).

7. Nishi, N., Takahashi, S., Matsumoto, M., Tanaka, A., Muraya, K., Takamuku, T. and Yamaguchi, T. "Hydrogen-bonded cluster formation and hydrophobic solute association inaqueous solutions of ethanol", The Journal of Physical Chemistry, 99(1), pp. 462-468 (1995).

8. Li, R, D’Agostino, C., McGregor, J., Mantle, M.D., Zeitler, J.A. and Gladden, L.F. "Mesoscopic structuring and dynamics of alcohol/water solutions probed by terahertz time-domain spectroscopy and pulsed field gradient nuclear magnetic resonance", The Journal of Physical Chemistry B, 118(34), pp. 10156-10166 (2014).

9. Puzenko, A., Hayashi, Y., Ryabov, Y.E., Balin, I., Feldman, Y., Kaatze, U. and Behrends, R. "Relaxation dynamics in glycerol-water mixtures: I. glycerol-rich mixtures", The Journal of Physical Chemistry B, 109(12), pp. 6031-6035 (2015).

10. Makoto, U., Harada, M., Tsukahara, T. and Okada, T. "Behavior of polyhydric alcohols at ice/liquid interface", The Journal of Physical Chemistry C, 117(47), pp. 24873-24882 (2013).

11. Weng, L., Chen, C., Zuo, J. and Li, W. "Molecular dynamics study of effects of temperature and concentration on hydrogen-bond abilities of ethylene glycol and glycerol: implications for cryopreservation", The Journal of Physical Chemistry A, 115(18), pp. 47294737 (2011).

12. Dlugoborski, T., Hawlicka, E. and Swiatla-Wojcik, D. "Effect of a solute on water properties-MD simulation studies", Journal of Molecular Liquids, 85(1), pp. 97104 (2000).

13. Corsaro, C., Spooren, J., Branca, C., Leone, N., Broccio, M., Kim, C., Chen, S.H., Stanley, H.E. and Mallamace, F. "Clustering dynamics in water/methanol mixtures: A nuclear magnetic resonance study at 205 $\mathrm{K}<\mathrm{T}<295 \mathrm{~K}$ ", The Journal of Physical Chemistry B, $112(34)$, pp. 10449-10454 (2008). 
14. Towey, J.J., Soper, A.K. and Dougan, L. "Preference for isolated water molecules in a concentrated glycerolwater mixture", The Journal of Physical Chemistry B, 115(24), pp. 7799-7807 (2011).

15. Cong, C., Li, W.Z., Song, Y.C. and Yang, J. "Hydrogen bonding analysis of glycerol aqueous solutions: A molecular dynamics simulation study", Journal of Molecular Liquids, 146(1), pp. 23-28 (2009).

16. Brand, T., Cabrita, E.J. and Berger, S. "Theory and application of NMR diffusion studies", In Modern Magnetic Resonance, pp. 135-143, Springer, Netherlands (2006).

17. Williams, J.W. and Cady, L.C. "Molecular diffusion in solution", Chemical Reviews, 14(2), pp. 171-217 (1934).

18. Hills, E.E., Abraham, M.H., Hersey, A. and Bevan, C.D. "Diffusion coefficients in ethanol and in water at 298K: Linear free energy relationships", Fluid Phase Equilibria, 303(1), pp. 45-55 (2011).

19. Chen, Q., Wang, Q., Liu, Y.C. and Wu, T. "The effect of hydrogen bonds on diffusion mechanism of water inside single-walled carbon nanotubes", The Journal of Chemical Physics, 140(21), pp. 214507 (2014).

20. D'Errico, G., Ortona, O., Capuano, F. and Vitagliano, V. "Diffusion coefficients for the binary system glycerol+ water at $25^{\circ} \mathrm{C}$. A velocity correlation study", Journal of Chemical \& Engineering Data, 49(6), pp. 1665-1670 (2004).

21. Chen, N. and Chan, T.C. "Experimental study of hydrogen bonding by mutual diffusion", Chem. Commun., 7, pp. 719-720 (1997).

22. Karmazina, T.V., Kavitskaya, A.A., Slisenko, V.I., Petrachkov, A.A. and Vasilkevich, A.A. "Changing of mechanisms of self-diffusion of water molecules under nanofiltration of electrolyte solution", Desalination, 184(1), pp. 337-345 (2005).

23. Almásy, L, Banki, P., Bellissent-Funel, M.C., Bokor, M., Cser, L., Jancsó, G., Tompa, K. and Zanotti. J.M. "QENS and NMR studies of 3-picoline-water solutions", Applied Physics A, 74(1), pp. s516-s518 (2002).

24. Mills, R. "Self-diffusion in normal and heavy water in the range 1-45", The Journal of Physical Chemistry, $\mathbf{7 7}(5)$, pp. $685-688$ (1973).

25. Masaro, L., Zhu, X.X. and Macdonald, P.M. "Selfdiffusion of oligo-and poly (ethylene glycol)s in poly (vinyl alcohol) aqueous solutions as studied by pulsedgradient NMR spectroscopy", Macromolecules, 31(12), pp. 3880-3885 (1998).

26. Johnson, C.S. "Diffusion ordered nuclear magnetic resonance spectroscopy: Principles and applications", Progress in Nuclear Magnetic Resonance Spectroscopy, 34(3), pp. 203-256 (1999).

27. Chen, C., Li, W.Z., Song, Y.C., Weng, L.D. and Zhang, N. "Concentration dependence of water selfdiffusion coefficients in dilute glycerol-water binary and glycerol-water-sodium chloride ternary solutions and the insights from hydrogen bonds", Molecular Physics, 110(5) pp. 283-291 (2012).

28. Egorov, A.V., Lyubartsev, A.P. and Laaksonen, A. "Molecular dynamics simulation study of glycerolwater liquid mixtures", The Journal of Physical Chemistry B, 115(49), pp. 14572-14581 (2011).

29. Chen, B., Sigmund, E.E. and Halperin, W.P. "StokesEinstein relation in supercooled aqueous solutions of glycerol", Physical Review Letters, 96(14), pp. 145502 (2006).

30. Wu, D., Chen, A. and Johnson, C.S. "An improved diffusion-ordered spectroscopy experiment incorporating bipolar-gradient pulses", Journal of Magnetic Resonance, Series A, 115(2), pp. 260-264 (1995).

31. Morris, K.F. and Johnson Jr, C.S. "Diffusion-ordered two-dimensional nuclear magnetic resonance spectroscopy", Journal of the American Chemical Society, 114(8), pp. 3139-3141 (1992).

32. Morris, G.A. "Diffusion-ordered spectroscopy", In Encyclopedia of Nuclear Magnetic Resonance, David M. Grant and Robin K. Harris, Eds., 9, pp. 35-44, John Wiley \& Sons, Ltd, Chichester (2002).

33. Holz, M. and Weingartner, H. "Calibration in accurate spin-echo self-diffusion measurements using ${ }^{1} \mathrm{H}$ and less-common nuclei", Journal of Magnetic Resonance, 92(1), pp. 115-125 (1991).

34. Price, W.S., Ide, H. and Arata, Y. "Solution dynamics in aqueous monohydric alcohol systems", The Journal of Physical Chemistry A, 107(24), pp. 4784-4789 (2003).

35. Xu, L., Mallamace, F., Yan, Z., Starr, F.W., Buldyrev, S.V. and Stanley, H.E. "Appearance of a fractional Stokes-Einstein relation in water and a structural interpretation of its onset", Nature Physics, 5(8), pp. 565-569 (2009).

36. Kholmirzo, K., Dushanov, E., Yasuoka, K., Khalil, H., Galal, A., Ahmed, S., Sweilam, N. and Moharram, H. "Molecular dynamics simulation of the interaction of ethanol-water mixture with a Pt surface", Natural Science, 3(12), pp. 1011-1021 (2011).

37. Zhang, N., Shen, Z., Chen, C., He, G. and Hao, C. "Effect of hydrogen bonding on self-diffusion in methanol/water liquid mixtures: A molecular dynamics simulation study", Journal of Molecular Liquids, 203, pp. 90-97 (2015).

38. Corsaro, C., Maisano, R., Mallamace, D. and Dugo, G. "NMR study of water/methanol solutions as a function of temperature and concentration", Physica A: Statistical Mechanics and Its Applications, 392(4), pp. 596-601 (2013).

\section{Biographies}

Elham Fadaei is currently a PhD student in Physical Chemistry at Sharif University of Technology and works in the field of NMR spectroscopy. She was 
awarded as a talented student in both master and bachelor's programs at Isfahan University of Technology in 2005-2011. She worked on Ion Mobility Spectrometry (IMS) as her master thesis.

Mohsen Tafazzoli received his PhD degree in 1992 from University of California at Davis, USA, under supervision of Professor N.S. True. Presently, he is a Professor of Physical Chemistry at Sharif University of Technology, Teheran, Iran. His research involves studies of intermolecular interactions, both in liquid and gas phases and calculation of NMR parameters. Also, he is interested in structure characterization of Carbohydrates. 